\title{
Microalbuminuria correlates with intestinal histopathological grading in patients with inflammatory bowel disease
}

\author{
N Mahmud, G S A McDonald, D Kelleher, D G Weir
}

\section{Abstract}

It has previously been shown that microalbuminuria is a useful disease activity marker for inflammatory bowel disease (IBD). Microalbuminuria correlates strongly with the markers of clinical and laboratory disease activity such as erythrocyte sedimentation rate (ESR), and C reactive protein (CRP). The aim of this study was to discover if microalbuminuria accurately reflects the intestinal inflammation by correlating it with intestinal inflammation using a standard histopathological grading system in patients with ulcerative colitis and Crohn's colitis. Forty two patients with IBD who had undergone endoscopic examination of the entire colon for the assessment of severity and extent of the disease (Crohn's colitis $(n=21)$, ulcerative colitis $(n=21))$ were recruited to the study. Patients with small bowel Crohn's disease were not studied. Twenty four patients had left sided colonic disease and 18 patients had extensive colonic disease. Each patient's colonic biopsy specimens were scored blindly by a histopathologist and a composite score was compiled on the basis of the severity of changes in the enterocytes and crypts and the cellularity of the lamina propria. A clinical disease activity was obtained using the simple index of Harvey and Bradshaw. Microalbuminuria was measured in all patients by an immunoturbiditimetric method. ESR and CRP were also measured, as indicators of acute phase response in the same patients. It was found that patients with active IBD had higher concentrations of microalbuminuria compared with those patients in remission (median $222 \mu \mathrm{g} / \mathrm{min}$ (range 40-686 $\mu \mathrm{g} / \mathrm{min}$ ) $v$ median $96 \mu \mathrm{g} / \mathrm{min}$ (range 30-376 $\mu \mathrm{g} / \mathrm{min}) ; p<0.001)$ ). Significantly higher concentrations of microalbuminuria were also detected in patients with extensive colonic IBD compared with those patients with left sided disease (median $297 \mu \mathrm{g} / \mathrm{min}$ (range 132-686 $\mu \mathrm{g} / \mathrm{min}$ ) $v$ median $101 \mu \mathrm{g} / \mathrm{min}$ (range $30-433 \mu \mathrm{g} / \mathrm{min}) ; \quad \mathbf{p}<0.001)$ ). A strong positive correlation was seen between microalbuminuria and intestinal histopathological score in IBD patient groups with left sided colitis $(r=0.77 ; p<0.001)$ and extensive disease $(r=0.71 ; p<0.01)$. The standard histopathological grading system correlated with the clinical disease activity $(r=0.64 ; p<0.005)$ and CRP $(r=0.62 ; p<0.02)$, however, it did not correlate with ESR. In conclusion, the strong correlation of microalbuminuria with a standard intestinal histopathological grading system suggests that microalbuminuria accurately reflects the severity of colonic inflammation in patients with Crohn's colitis and ulcerative colitis.

(Gut 1996; 38: 99-103)

Keywords: microalbuminuria, inflammatory bowel disease, ulcerative colitis, Crohn's disease, disease activity, intestinal histopathological grading.

The aetiology and pathogenesis of chronic inflammatory bowel disease (IBD) remain unknown. There are many different methods of assessing disease activity including various clinical scoring systems that heavily rely on subjective indices. Laboratory tests, including the measurement of acute phase proteins, offer more objective assessment. ${ }^{12}$ Acute phase proteins, however, are likely to be changed by treatments such as corticosteroid or immunosuppressive agents. Gastrointestinal protein loss also offers an objective method, ${ }^{34}$ however, stool collection and processing for measurement of protein is cumbersome. Indium labelled leucocyte scanning has been found to correlate well with the clinical activity, colonoscopy, and histological assessment of disease activity. However, this is expensive, time consuming, and results in significant exposure to radioactivity. ${ }^{5-7}$ Colonic mucosal histology obtained from biopsy specimens is probably the most accurate objective method of assessing the true disease activity in IBD. Endoscopic examination with biopsy is invasive and expensive, however, and is contraindicated in acutely ill patients with severe disease. Thus there is a need for a simple objective test, which will permit serial measurements of the progress of the disease and an evaluation of the response to treatment, which can accurately reflect the degree of intestinal inflammation.

Persistently increased urinary excretion of albumin above normal, which is not detected by the semiquantitative dipstrip test is defined as microalbuminuria. Albuminuria excretion greater than 20 micrograms per minute in an overnight urine specimen is diagnostic of microalbuminuria. Microalbuminuria is also described as a non-specific marker for acute illness (including myocardial infarction) ${ }^{8}$ and other conditions such as, septicaemia, 
(A) Histological assessment of mucosal biopsy specimens

\begin{tabular}{ll}
\hline Enterocytes & \\
Normal & 0 \\
Loss of single cell & 1 \\
Loss of groups of cells & 2 \\
Frank ulceration & 3 \\
Crypts & 0 \\
Normal & \\
Single inflammatory cells & 1 \\
Cryptitis & 2 \\
Crypt abscesses & 3 \\
Lamina propria & \\
Mononuclear cells & 0 \\
Normal & 1 \\
Slight increase & 2 \\
Moderate increase & 2 \\
Marked increase & 3 \\
Neutrophils & \\
Normal & 0 \\
Slight increase & 1 \\
Moderate increase & 2 \\
Marked increase & 3
\end{tabular}

(B) Conversion of histological scores to grades

\begin{tabular}{ll}
\hline Grade & Total score \\
\hline 0 & $0-1$ \\
1 & $2-4$ \\
2 & $5-8$ \\
3 & $8-12$ \\
\hline
\end{tabular}

post-trauma, and burns. ${ }^{9}$ The microalbuminuria in these conditions most probably occurs as a result of the acute phase response to inflammatory mediators and in this respect it may provide a more longstanding and easily measurable indicator than other acute phase parameters. We have previously shown that microalbuminuria is a useful disease activity marker for inflammatory bowel disease. ${ }^{10}$ Significantly higher levels of microalbuminuria were detected in IBD patients with active disease compared with those with inactive disease and controls. Microalbuminuria correlated strongly with markers of clinical and laboratory disease activity such as erythrocyte sedimentation rate (ESR) and $C$ reactive protein (CRP). The aim of this study was to find out if microalbuminuria accurately reflects the severity of intestinal inflammation using a standard histopathological grading system ${ }^{5}$ in patients with inflammatory bowel disease.

\section{Methods}

Forty two patients (male; $\mathbf{n}=19$; median age 40 years; range 18-69 and female; $n=23$; median age 37 years; range 18-68) with IBD (ulcerative colitis 21 and Crohn's disease 21) undergoing full colonoscopic assessment were studied. The median duration of the disease was 8.4 years and ranged from one week to 23 years. At the time of colonoscopic assessment all except 10 patients were receiving 5-ASA maintenance treatment (olsalazine $=18$; sulphasalazine $=11$; mesalazine $=3$ ) and two patients were receiving concomitant corticosteroid treatment. These patients did not have radiological evidence of small bowel disease. Multiple mucosal biopsy specimens were taken from the macroscopically affected regions to establish the severity of the disease activity. Each biopsy specimen was assessed blindly by one histopathologist with a special interest in gastrointestinal diseases and who was unaware of the disease activity index of each case. In each biopsy specimen, scores were assigned to the severity of surface enterocyte damage, cryptitis, and acute inflammation and chronic inflammation in the lamina propria. ${ }^{5}$ The mean of these scores was obtained and converted to a grade 5 (Table). Twenty four patients had left sided colonic disease and 18 patients had extensive colonic disease. The second group having disease extending at least to the hepatic flexure. On the day of endoscopy, clinical disease activity was quantified using the simple index of Harvey and Bradshaw (HBI) ${ }^{11}$ and all patients had venesection for routine biochemistry and for the estimation of CRP and ESR. Disease was considered active if the HBI was 4 or greater.

A 12 hour overnight urine collection was obtained from all patients from $9 \mathrm{pm}$ to $9 \mathrm{am}$, the urine collected was received in the laboratory within two hours, and the volume measured. Thirty millilitres of this urine was then stored at $-20^{\circ} \mathrm{C}$ for measurement of microalbuminuria. In addition routine urine analysis was performed for the estimation of urinary urea, electrolytes, protein, and creatinine clearance.

\section{Exclusion criteria}

Patients with diabetes mellitus, hypertension, renal pathology, urinary tract infection, and patients using diuretics, non-steroidal antiinflammatory agents, and age more than 70 years were excluded.

\section{Microalbuminuria and CRP}

Microalbuminuria was measured in all patients and controls using the immunoturbiditimetric method (Microalbs, AMES, Bucks, UK). In this technique human albumin reacts with a specific antibody in the presence of polyethylene glycol. As the antibody was present in large excess the precipitate forms a complex related to the concentration of albumin in the sample. The turbidity was photometrically measured at wavelength $340 \mathrm{~nm}$. Coefficient of variance was less than 3\%. CRP was measured by nephelometry.

\section{Statistical analysis}

Statistical comparison was carried out using the Mann-Whitney U test. Simple regression analysis was used to calculate correlation coefficients for parametric data and Spearman's rank correlation coefficient was used for non-parametric data. Results are expressed as median (range) and a probability value of $<0.05$ was considered to be statistically significant.

\section{Results}

\section{Microalbuminuria in IBD}

The concentrations of microalbuminuria were significantly higher in patients with active IBD compared with those patients in remission (median 222 (range 40-686) $\mu \mathrm{g} / \mathrm{min} v 96$ (30-376) $\mu \mathrm{g} / \mathrm{min} ; \mathrm{p}<0.001$; reference ranges $0-20 \mu \mathrm{g} / \mathrm{min}$; Fig 1). Significantly higher concentrations of microalbuminuria were also detected in patients with extensive colonic IBD compared with those patients with rectosigmoid disease (median $297 \mu \mathrm{g} / \mathrm{min}$ (range $132-686 \mu \mathrm{g} / \mathrm{min})$ v $101(30-433) ; \mathrm{p}<0.001)$; Fig 2). There was no significant difference, however, in concentrations of microalbuminuria in patients with ulcerative colitis and those patients with Crohn's disease (median $145 \mu \mathrm{g} / \mathrm{min}$ (range 40-423) v 216 (30-686); $\mathrm{p}=0 \cdot 31$ ).

\section{Correlation of microalbuminuria, \\ histopathological grading, CRP, ESR, and clinical disease activity}

Microalbuminuria correlated significantly with intestinal histopathological grading in IBD patients with rectosigmoid disease $(r=0.77$; $\mathrm{p}<0.001)$ and extensive disease $(r=0.71$; $\mathrm{p}<0.01$; fig 3 ). Microalbuminuria also correlated significantly with the clinical disease activity of patients with left sided colonic disease $(r=0.63 ; \mathrm{p}<0.002)$ and those with extensive colonic disease $(r=0.62 ; \mathrm{p}<0.03)$. A strong correlation was also seen between 


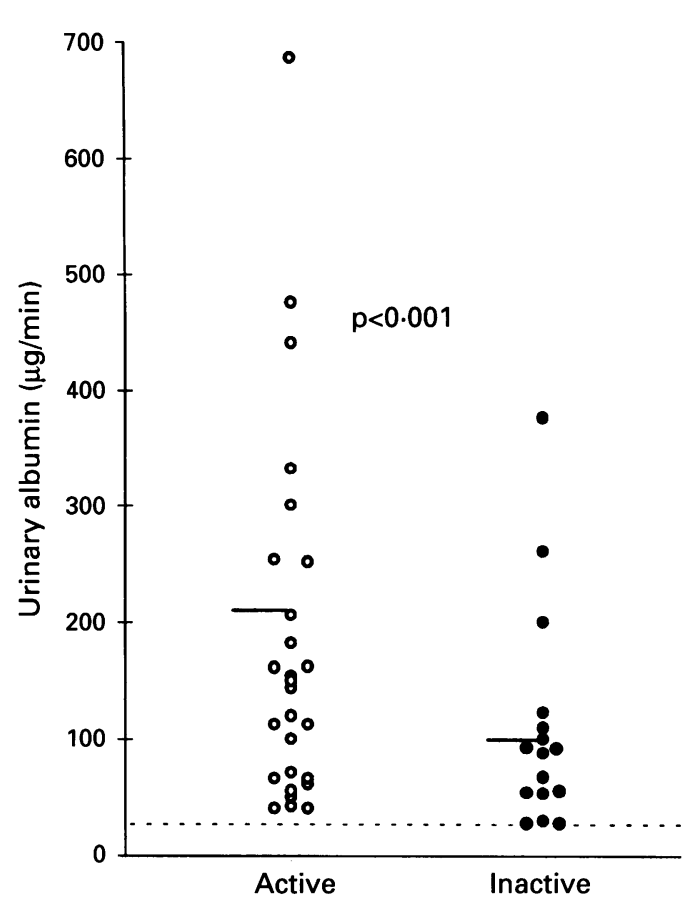

Figure 1: Urinary albumin in IBD. Each point represents a single patient and the mean of a duplicate measurement. The median is shown by a horizontal line. The upper normal range for urinary albumin is shown by the dotted line. p Value, calculated using the Mann-Whitney $U$ test, refers to comparison with inactive disease.

microalbuminuria and CRP $(r=0 \cdot 80$; $\mathrm{p}<0.001)$. No significant correlation was seen, however, between microalbuminuria and ESR $(r=0 \cdot 1 ; \mathrm{p}=0 \cdot 20)$.

The histopathological grading showed a strong positive correlation with the clinical disease activity $(r=0.64 ; \mathrm{p}<0.005)$ and CRP $(r=0.62 ; \mathrm{p}<0.02)$, however, it did not correlate with ESR. The clinical disease activity assessed by HBI correlated significantly with CRP $(r=0.478 ; \mathrm{p}<0.03)$. However, in this study HBI did not correlate with ESR $(r=0.297 ; \mathrm{p}=0.45)$.

There was no significant difference in serum creatinine concentrations in patients with histopathological grade 0 compared with those with intestinal histopathological grade 1, 2, 3 (grade 0 (median 79; range 62-98) $v$ grade 1 (median 78; range 68-110); grade 2 (median 86 ; range $72-114$ ); grade 3 (median 82 ; range 65-106); reference range 50-125 $\mu \mathrm{mol} / \mathrm{l})$.

Urine analysis, urinary urea/electrolytes, and creatinine clearance were normal in all patients.

\section{Discussion}

The evaluation of IBD activity has mainly been based on clinical indices, especially in controlled therapeutic trials. The search for more objective disease activity markers had led to a profusion of published studies, ${ }^{1-46712-14}$ which have shown little consensus. Indeed the evaluation of serological and biochemical indices of disease activity has largely been based on correlations with clinical scores such as the Crohn's disease activity index ${ }^{15}$ or the HarveyBradshaw score. ${ }^{11}$ We have recently evaluated microalbuminuria as a disease activity index in IBD. ${ }^{10}$ Interestingly, while microalbuminuria concentrations correlated significantly with clinical disease activity scores, values of microalbuminuria rarely returned completely to normal in patients with chronic IBD. Hence, the high concentrations of microalbuminuria could reflect ongoing low grade inflammation in the diseased intestine even while in clinical remission. From our previous study, we have concluded that microalbuminuria does not result from glomerular or tubular damage as a result of drugs (sulphasalazine, olsalazine, mesalazine), as there was no correlation with drug treatment in patients with active disease.

Furthermore, there was no indication of drug induced tubular damage; a sensitive index of tubular dysfunction glutathione $S$ transferase pi was measured and found to be negative in all cases tested. ${ }^{10}$

While endoscopy with biopsies is the gold standard for the evaluation of inflammatory activity in patients with IBD, its use in the ongoing assessment of disease activity is limited by its invasiveness, cost, and inconvenience. Comparatively few studies have considered the question of whether indices of disease activity correlate with histological parameters of disease. In one study there was no significant correlation between clinical disease activity assessed by CDAI and by endoscopic parameters. ${ }^{12} \mathrm{~A}$ further study by Gomes et al found no significant correlation between the index score and histological score for Crohn's colitis, or either the index score and a macroscopic or microscopic score in ulcerative colitis. No significant correlation of microscopic score was found with CRP, ESR, white blood count, platelet count or albumin concentration in patients with either ulcerative colitis or Crohn's colitis. ${ }^{13}$ In our study, however, both CRP and activity indices were found to correlate significantly with the histological score. This may reflect the fact that only two of our patients were receiving corticosteroids at

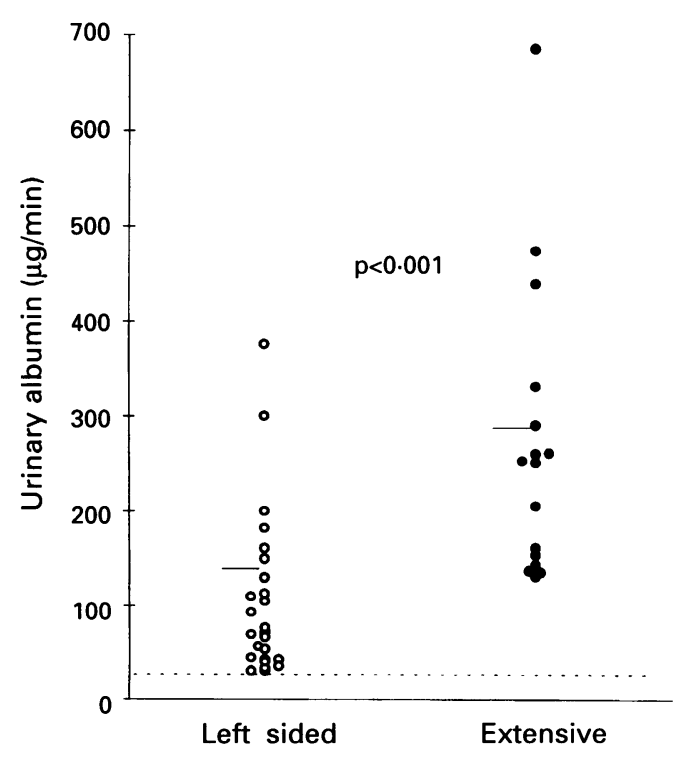

Figure 2: Urinary albumin in IBD. Each point represents a single patient and the mean of a duplicate measurement. The median is shown by a horizontal line. The upper normal range for urinary albumin is shown by the dotted line. $p$ Values, calculated using the Mann-Whitney U test, refer to comparison with left sided disease. 


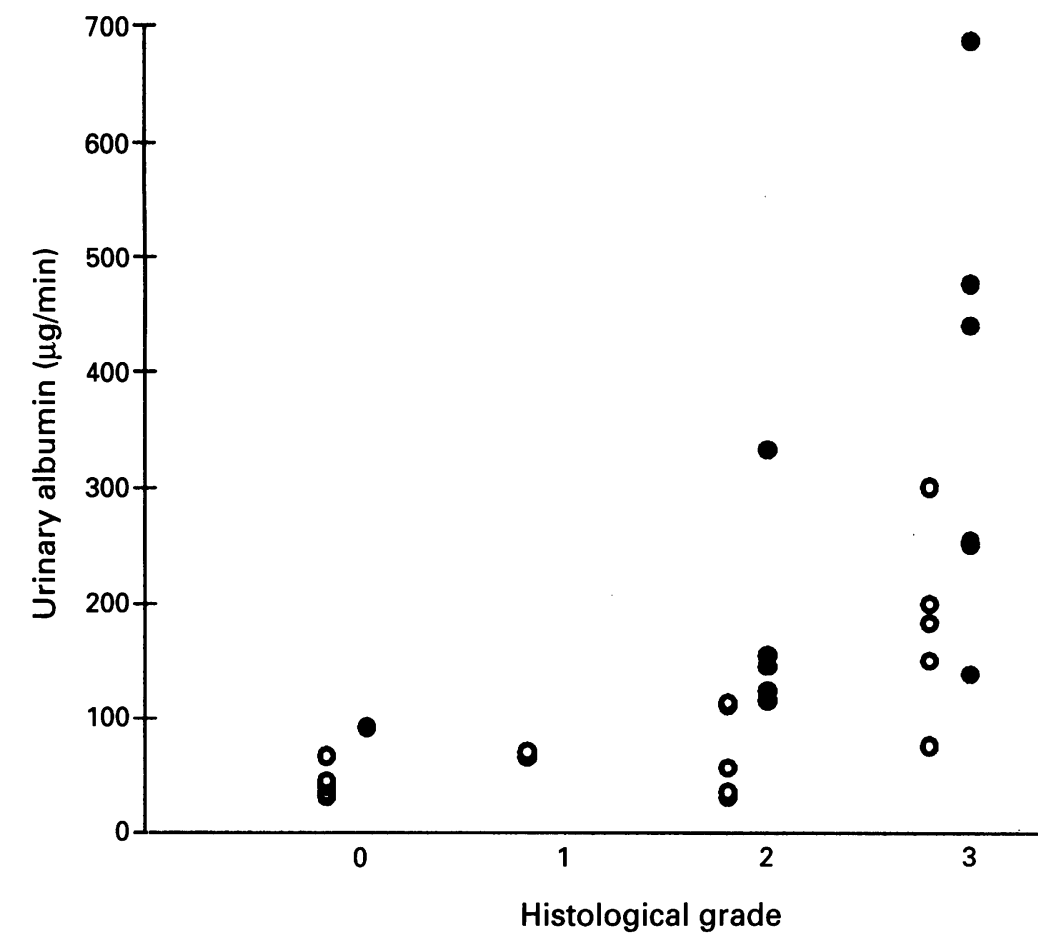

Figure 3: Urinary albumin by histological grade in IBD. Each point represents a single patient. Spearman's rank correlation coefficient, $\mathrm{r}$, for left sided disease (O) is 0.77 and for extensive disease (๑) is $0 \cdot 71$.

the time of study. Histological activity has been found to correlate with indium 111-labelled leucocyte excretion. ${ }^{5}$ This is an expensive time consuming procedure, however, which exposes the subject to radiation. Accordingly, this may not be suitable for frequent monitoring of disease activity particularly when evaluating different treatments. Park and coworkers using indium scanning correctly determined the extent of disease in only $58 \%$ of the patients and there was a poor correlation between the scan scores and the indices of disease activity including clinical scores, ESR, CRP, and gastrointestinal protein loss. ${ }^{14}$

Disease extent and disease activity are two important assessments that influence the treatment of IBD. In this study, we have evaluated microalbuminuria as an indicator of disease activity in patients with IBD. Microalbuminuria showed a significant correlation with histological indices of disease activity and also with extent of disease. Furthermore, our data show that both clinical disease activity and CRP also correlate with histological grading. In this study we did not recruit patients with small bowel Crohn's disease, as endoscopy cannot assess the extent of small bowel involvement and histology is difficult to obtain, hence these results cannot be assumed to be valid for small bowel disease.

The pathogenesis of microalbuminuria could be related to inflammatory changes in the intestine. The histological picture of IBD is marked by the presence of inflammatory cells (neutrophils, lymphocytes, and histocytes) in the intestinal mucosa. The activated inflammatory cells may contribute to tissue damage at the sites of inflammation. Multiple nucleated cells including macrophages and circulating blood monocytes are capable of producing a number of cytokine mediators such as interleukin 1 (IL 1 ), tumour necrosis factor $\alpha$ (TNF $\alpha$ ), and IL 6. High concentrations of circulating $\mathrm{TNF} \alpha$, peripheral mononuclear leucocytes IL $1 \beta$ and IL 2 production, and colonic mucosal IL 6 have been described in patients with active IBD. ${ }^{16-19}$ Though, circulating TNF may be responsible for some of the systemic reactions in IBD. There is evidence that hypoalbuminaemia found in chronic IBD results mainly from loss of albumin into the lumen. ${ }^{3}$ This may reflect TNF induced disruption of mucosal sulphated glycosaminoglycans. ${ }^{20}$ Microalbuminuria in IBD may similarly reflect the effects of circulating cytokines such as TNF on glycosaminoglycans in the renal microvasculature. Experimental enzymatic digestion of glycosaminoglycans results in increased vascular permeability for albumin. ${ }^{21-24}$ There is evidence that macrophage derived cytokines IL 1 and TNF $\alpha$ can inhibit the synthesis of glycosaminoglycans and enhance their loss from cultured endothelial cells. ${ }^{2425}$ We have recently shown that microalbuminuria correlates with serum TNF concentrations. ${ }^{26}$ Hence both experimental data and clinical data suggest that microalbuminuria in IBD could be induced by cytokines produced by inflammatory cells in the intestine causing glycosaminoglycans disruption.

The development of a simple, inexpensive, and non-invasive index that accurately reflects histological disease activity in IBD is clearly of importance in monitoring the progress of a patient's illness. Clinical studies of treatment for IBD have hitherto been based largely on clinical indicators of disease activity. In many instances these indicators are not sufficiently sensitive to reflect low grade ongoing inflammation in the intestine. The design of clinical trials with an end point of endoscopic or histological remission could provide an alternative approach for the assessment of new treatments for chronic IBD. Our data suggest that microalbuminuria may provide an additional non-invasive indicator of colonic inflammation in patients with chronic IBD.

Dr Dermot Kelleher is a Wellcome Senior Fellow in Clinical Science. These data have been previously published in abstract form in Gastroenterology 1994; 106: A726.

1 Beck IT. Laboratory assessment of IBD. Dig Dis Sci 1987; 32 (suppl): 26-41.

2 Chambers RE, Stross P, Barry RE, Whicher JT. Serum amyloid-A protein compared with C-reactive protein $\alpha 1-$ antichymotrypsin and $\alpha 1$-acid glycoprotein as a monitor of inflammatory bowel disease. Eur f Clin Invest 1987; 17: 460-4.

3 Becken WL, Busch HJ, Sylwester DL. Intestinal protein loss in Crohn's disease. Gastroenterology 1972; 62: 207-15.

4 von Tongeren JH, Eekhout A. Criteria to assess the severity of Crohn's disease and effect of treatment. In: Weterman IT, Pena AS, Booth CC eds. The management of Crohn's disease. Pena AS, Booth CC, eds. The management of Crohn's disease. Proceedings of the Workshop on Crohn's disease. Leyden

23-25 October 1975. Amsterdam: Elsevier, 1976: 153-8. averymuttu SH, Camerilli M, Rees H, Lavender JP, Hodgson HJF, Chadwick VS. Indium-111 granulocyte scanning in the assessment of disease extent and disease activity in inflammatory bowel disease. Gastroenterology 1986; 90: 1121-8.

6 Stein DT, Gray GM, Gregory PB, Anderson M, Goodwin DA, McDougall IR. Location and activity of ulcerative colitis and Crohn's colitis by Indium-111 leucocyte scan. Gastroenterology 1983; 84: 388-93.

7 Saverymuttu SH, Peters AM, Lavender JP, Hodgson HJF, Chadwick VS. Quantitative faecal Indium-111 labelled leucocyte excretion in the assessment of disease in Crohn's disease. Gastroenterology 1983; 85: 1333-9. 
8 Gosling $P$, Shearman CP. Increased levels of urinary proteins: marker of vascular permeability? Ann Clin Biochem 1988; 25 (suppl): 150-1.

9 Gosling P, Sutcliffe AJ, Cooper MACS, Jones AF. Burns and trauma associated proteinuria: role of lipid peroxidaand trauma associated proteinuria: role of lipid peroxidation, ren

10 Mahmud N, Stinson J, O'Connell M, Mantle T, Keeling $\mathrm{PWN}$, Feely $\mathrm{J}$, et al. Microalbuminuria in patients with inflammatory bowel disease. Gut 1994; 35: 1599-604.

11 Harvey RF, Bradshaw JW. A simple index of Crohn's disease activity. Lancet 1980; i: 514.

12 Cellier C, Sahmoud T, Froguel E, Adenis A, Belaiche J, Bretagne J-F, et al Correlation between clinical activity, Bretagne J-F, endoscopic severtic or ileocolonic Crohn's disease. A prospect 35: 231-5.

13 Gomes P, Boulay Claire Du, Smith CL, Holdstock G. Relationship between disease activity indices and colonoscopic findings in patients with colonic inflammatory bowel disease. Gut 1986; 27: 92-5.

14 Park RHR, McKillop JH, Duncan A, MacKenzie JF, Russell RI. Can Indium-111 autologous mixed leucocyte scanning accurately assess disease extent and activity in Crohn's disease? Gut 1988; 29: 821-5.

15 Best WR, Becklet JM, Singleton JW, Kern F. Development of a Crohn's disease activity index: National Co-operative Crohn's disease study. Gastroenterology 1976; 70: 439-44.

16 Nathan CF. Secretory products of macrophages. 7 Clin Invest 1987; 79: 319-26.

17 Murch SH, Lamkin VA, Savage MO, Walker-Smith JA, MacDonald TT. Serum concentration of TNFa in childhood chronic inflammatory bowel disease. Gut 1991; 32: 913-7.

18 Nakamura M, Saito H, Kananuki J, Tamura Y, Yoshida S.
Cytokine production in patients with inflammatory bowel disease. Gut 1992; 33: 933-7.

19 Mitsuyama K, Sasaki E, Toyonaga A, Ikeda H, Tsuruta O, Irie $A$, et al. Colonic mucosal interleukin-6 in inflammaIrie A, et al. Colonic mucosal interleukin-6 in

20 Murch SH, MacDonald TT, Walter-Smith JA, Levin M, Lionetti P, Klein NJ. Disruption of sulphated glycosaminoglycans in intestinal inflammation. Lancet 1993; 341: 711-4.

21 Rosenzweig LJ, Kanwar YS. Removal of sulphated (heparan sulphate) and non-sulphated (hyaluronic acid) results in increased permeability of the glomerulus basement membrane to 125I-bovine serum albumin Lab Invest 1982; 47: 177-84.

22 Kanwar YS, Linker A, Farquar MG. Increased permeability of glomeruluar basement membrane to ferritin after removal of glycosaminoglycans (heparan sulphate) by removal of glycosaminoglycans (heparan sulphate) by enzymatic digestion. $\mathcal{F}$ Cell Biol 1980; 86: 688-94.

23 Vehaskari VM, Chang CT-C, Stevens JK, Robson AM. The effects of polycations on vascular permeability in the rats. f Clin Invest 1984; 84: 1053-9.

24 Klein NJ, Shenan GI, Heyderman RS, Levin M. Alteration in glycosaminoglycan metabolism and surface charge on human umbilical vein endothelial cells induced by cytokines, endotoxin and neutrophils. 7 Cell Sci 1992; 102: 821-32.

25 Yi ES, Ulich TR. Endotoxin, interleukin-1, and tumour necrosis factor cause neutrophil-dependent microvascular leakage in postcapillary venules. Am f Pathol 1992; 140: leakage

26 Mahmud N, O'Connell MA, Stinson J, Goggins MG, Weir DG, Kelleher D. Tumour necrosis factor- $\alpha$ and microalbuminuria in patients with inflammatory bowel disease. European fournal of Gastroenterology and Hepatology 1995; 7: 215-9. 